\title{
Pacientes ilegítimos: Acceso a la salud de los inmigrantes indocumentados en Chile
}

\author{
Illegitimate patients: Undocumented immigrants' \\ access to health care in Chile
}

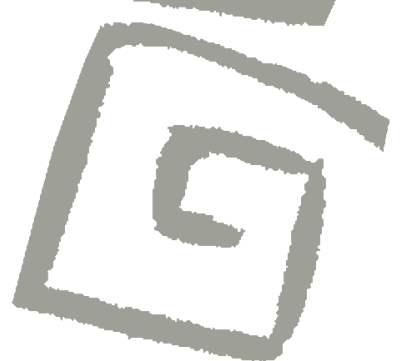

Nanette Liberona Concha ${ }^{1}$, Miguel Ángel Mansilla ${ }^{2}$

${ }^{1}$ Antropóloga. Investigadora, Instituto de Estudios Internacionales, Universidad Arturo Prat, Chile. $\triangle$ (iD)

${ }^{2}$ Sociólogo. Investigador Instituto de Estudios Internacionales, Universidad Arturo Prat, Chile. $\bowtie$ (iD)
RESUMEN Chile se ha ido convirtiendo en un país de destino para las migraciones sudamericanas, las cuales generan un impacto en los servicios públicos, en particular en salud, a nivel económico, social y cultural. El objetivo de este artículo es aportar información documentada sobre los problemas de acceso a la salud de los inmigrantes indocumentados. Trabajamos desde una metodología cualitativa, basada principalmente en una etnografía del espacio clínico. Para el análisis de los resultados nos hemos basado en las teorías de las relaciones asimétricas de poder, así como en las de las relaciones interétnicas. En los resultados de la investigación, se destaca el incumplimiento de la normativa y el ejercicio del criterio personal discrecional como barreras en el acceso. Concluimos que, en Chile, los inmigrantes en general e indocumentados, en particular, son considerados pacientes ilegítimos.

PALABRAS CLAVES Acceso a la Salud; Inmigración; Derechos del Paciente; Factores Socioeconómicos; Chile.

ABSTRACT In recent decades, Chile has become a destination for immigrants from other South American countries, which has significantly impacted public services - particularly the public health system - at the economic, social, and cultural levels. The aim of this paper is to provide substantiated information on issues concerning undocumented immigrants' access to health care in Chile. A qualitative methodology, fundamentally an ethnography of the clinical setting, was used. Results were then analyzed in relation to theories of power asymmetries and interethnic relations. The research results highlight the lack of compliance with existing regulations and the exercise of discretionary personal judgment as barriers to access. It is concluded that in Chile immigrants in general, and undocumented immigrants in particular, are considered to be illegitimate patients.

KEY WORDS Health Services Accessibility; Immigration; Patient Rights; Socioeconomics Factors; Chile. 


\section{INTRODUCCIÓN}

Esta investigación surge de la necesidad de responder a una serie de alertas con respecto a la vulneración del derecho a la salud de la población inmigrante en Chile, derecho definido en el marco jurídico chileno. Asimismo, de acuerdo a la última Encuesta de Caracterización Socioeconómica Nacional, del total de personas nacidas en el extranjero residentes en Chile, el 8,9\% no pertenece a ningún sistema previsional de salud, mientras que en la población chilena ese porcentaje llega al 2,5\%(1). Esto demuestra la desigualdad existente, en cuanto a previsión, entre la población nacional y extranjera.

Hace dos décadas que Chile viene constituyéndose en destino de migraciones sudamericanas ${ }^{(2)}$. El incremento de esta población ha significado un desafío mayor para las políticas públicas, en particular, en materia de salud. Las cifras entregadas por el Departamento de Extranjería y Migración en 2016 indican que, en los últimos 13 años, la presencia de ciudadanos extranjeros ha aumentado un $123 \%$. Es así como la población extranjera con residencia en Chile, para el 2014, se elevó a 410.988 personas, de las cuales la gran mayoría proviene de países limítrofes, siendo de origen peruano el 37,8\% del total de extranjeros, seguidos por los argentinos que constituyen el $16,3 \%$ y por los bolivianos que representan un $8,8 \%{ }^{(3)}$.

Con relación a la distribución, las regiones de Chile que han tenido el mayor crecimiento de población inmigrante se concentran en el norte del país, sobre todo en Antofagasta, Tarapacá y Atacama (Tabla 1). La región bifronteriza de Arica y Parinacota

Tabla 1. Número estimado de residentes extranjeros por región. Chile, 2009 y 2014.

\begin{tabular}{lccc} 
Región & $\begin{array}{c}\text { Residentes } \\
2009\end{array}$ & $\begin{array}{c}\text { Residentes } \\
2014\end{array}$ & $\begin{array}{c}\text { Crecimiento promedio } \\
\text { periodo (\%) }\end{array}$ \\
\hline XV de Arica y Parinacota & 10.383 & 14.577 & 7,03 \\
I de Tarapacá & 17.517 & 27.565 & 9,56 \\
II de Antofagasta & 18.200 & 30.829 & 11,19 \\
III de Atacama & 2.191 & 3.782 & 11,57 \\
IV de Coquimbo & 4.814 & 6.468 & 6,11 \\
V de Valparaíso & 20.781 & 24.934 & 3,71 \\
VI de O'Higgins & 4.358 & 5.704 & 5,54 \\
VII del Maule & 4.164 & 5.106 & 4,16 \\
VIII del Bio-Bio & 9.461 & 11.576 & 4,12 \\
IX de la Araucanía & 8.488 & 9.353 & 1,96 \\
X de los Lagos & 7.190 & 8.344 & 3,02 \\
XIV de Los Ríos & 2.840 & 3.206 & 2,45 \\
XI Aysén & 1.636 & 1.825 & 2,21 \\
XII de Magallanes & 2.349 & 2.986 & 4,92 \\
XIII Región Metropolitana & 202.685 & 285.274 & 7,11 \\
Total & 317.057 & 441.529 & 6,87
\end{tabular}

Fuente: Departamento de Extranjería y Migraciones, 2014. 
ha tenido un crecimiento promedio similar a la Región Metropolitana, que concentra el mayor número de inmigrantes ${ }^{(4)}$. Esta situación genera un impacto significativo en los servicios públicos de las regiones con mayor concentración de inmigrantes, especialmente en el Sistema Público de Salud (SPS). Además, hay que considerar que un porcentaje difícilmente cuantificable de la población inmigrante se encuentra en el país de forma irregular, son los llamados "indocumentados" o "irregulares". A nivel internacional "las estimaciones sugieren que tan solo entre 10 y $15 \%$ de las poblaciones migrantes están en un estatus 'irregular' en algún momento del tiempo"(5).

Ante esta situación, y en el marco de la nueva política migratoria, decretada en el año 2008 por la Presidenta de la República ${ }^{(6)}$, a través del Instructivo No. 9 en el que Chile se declara "país de acogida", el Ministerio de Salud (MINSAL) y el Fondo Nacional de Salud (FONASA) asumieron como marco ético la Convención Internacional sobre la Protección de los Derechos de Todos los Trabajadores Migratorios y sus Familiares, de las Naciones Unidas ${ }^{(7)}$. En su Tercera Parte, el artículo 28 establece que ellos:

...tienen derecho a recibir toda la atención médica necesaria en caso de urgencia para preservar su vida o evitar un daño irreparable a su salud, en base a la igualdad de tratamiento con las personas originarias del Estado en causa. No se les puede rechazar tal atención médica de urgencia por motivo de cualquier tipo de irregularidad en materia de estadía o de empleo. $^{(7)}$

Además de la atención de urgencia, el Estado de Chile decidió tomar en consideración a los "irregulares", firmando acuerdos interinstitucionales entre los Ministerios de Interior y de Salud. Así, se plasmó en la Resolución exenta No. 512 del MINSAL, la Resolución exenta No. 1914 del Ministerio del Interior, y el Oficio ORD-A-14 No. 3229 del MINSAL. Se extiende de esta manera el derecho a la previsión pública (FONASA) y a la atención médica a ciertas categorías de inmigrantes "irregulares" o indocumentados:

- Refugiados y solicitantes de asilo: otorga el derecho a previsión pública.

- Extranjeros menores en condición migratoria irregular: ordena la atención de salud y permite la tramitación de una visa temporaria.

- Mujeres embarazadas en situación migratoria irregular: ordena el control del embarazo y permite la tramitación de una visa temporaria.

La aplicación de estos acuerdos es lo que analizaremos en este manuscrito, a partir del trabajo etnográfico que hicimos en el espacio clínico y que en esta ocasión analizaremos a partir de las fuentes institucionales, de las percepciones de funcionarios del sector salud y desde una revisión de las notas publicadas por la prensa escrita. El punto de vista de los usuarios o pacientes inmigrantes no lo consideramos en este manuscrito por falta de espacio y porque lo hemos analizado en otras publicaciones ${ }^{(8,9)}$.

\section{Revisión de la bibliografía}

La relación entre migración y salud ha sido un tema de interés a nivel internacional, principalmente por organismos tales como la Organización de las Naciones Unidas (ONU), la Organización Internacional para las Migraciones (OIM) y la Organización Mundial de la Salud (OMS). Esta última se ha enfocado, entre otros, en las repercusiones de la migración internacional en la salud de los que se quedan atrás y de los que se desplazan ${ }^{(10)}$. EI sida y la salud sexual y reproductiva han sido temas de especial interés para los estudios sobre migración y salud pública ${ }^{(11)}$, así como la tuberculosis ${ }^{(12)}$. El informe Sida y movilidad plantea que las personas con $\mathrm{VIH}$ migran para tener acceso a tratamientos, y revela la polémica de que en algunos países se requiere una prueba obligatoria que determina la autorización de ingreso al país de acogida ${ }^{(13)}$. El principal enfoque de estos estudios es la consideración de que tanto las personas como 
las enfermedades traspasan las fronteras nacionales a pesar de los límites político-administrativos, estableciendo comparaciones epidemiológicas entre la población local y la población migrante. Los comportamientos de riesgo de los migrantes son una de las principales variables de análisis. Destacan los casos en zonas fronterizas como Centroamérica-México y México-EE.UU., pero también España, como lugares donde la población migrante es especialmente vulnerable y se ve afectada por enfermedades infectocotagiosas tanto por el estatus de salud en los países de origen, como por las condiciones de hacinamiento y pobreza en el país de acogida. Se desarrollan, por tanto, investigaciones sobre la protección social en salud, en las cuales el tema de las finanzas aparece como una dificultad a superar en el caso de la movilidad de México a EE.UU. ${ }^{(14)}$.

En Sudamérica, el enfoque se ha centrado en el acceso y uso de servicios de salud. En Argentina, un equipo dirigido por Jelin plantea: "la escasez de recursos coloca en el centro del debate la cuestión de cómo distribuirlos y si deben o no tener prioridad quienes residen en el país o en áreas específicas" $^{\prime \prime 15)}$. Es así como los derechos de los/as extranjeros/as son puestos en discusión en relación con los derechos de acceso a la salud de lo/as ciudadano/as locales o nativo/as. En el caso de estudio argentino se observa que las políticas de acceso a la salud dependen de la orientación y actitud de los directivos de las instituciones o de los propios médicos y personal de salud ${ }^{(16)}$, a pesar de que el acceso a la salud pública está garantizado por el Estado. En Brasil, por otra parte, la inmigración boliviana ha sido objeto de múltiples estudios, entre los cuales se incluye el tema del derecho a la salud, evidenciando discriminaciones y malos tratos dirigidos hacia esta comunidad, sin embargo, se concluye que las causas estarían más bien en una deficiencia estructural del sistema, ya que habría pacientes brasileños al margen de la sociedad que también recibirían tratos semejantes $^{(17)}$. Asimismo, Brasil ha vivido una fuerte demanda en salud, en las regiones fronterizas con Argentina, Paraguay y Uruguay, debido a los acuerdos de libre tránsito de personas entre los Estados partes del Mercado Común del Sur (MERCOSUR), que han permitido la libre circulación de personas, facilitando su residencia y permitiéndoles ejercer su derecho a la salud en este país receptor. Se concluye que los flujos migratorios en las fronteras develan las asimetrías en los sistemas de salud de los distintos estados, pero se cuestiona que se ha priorizado la dimensión económica por sobre el proceso de integración social del MERCOSUR ${ }^{(18)}$.

En Chile, desde el sector salud se han realizado algunos estudios que dan cuenta cómo esta problemática se ha convertido en tema de interés nacional. Es importante destacar los informes sobre salud y salud mental de inmigrantes de la zona norte de la Región Metropolitana ${ }^{(19)}$. Estos diagnósticos se acercan a la problemática desde las experiencias locales, y se establece la caracterización de esta nueva situación de salud dada por la inmigración. De igual forma, Vásquez-De Kartzow $^{(20)}$ reflexiona desde la pediatría para plantear que la migración en Chile implica retos para los cuales la sociedad chilena no está preparada, presentando los impactos que la migración ha generado a nivel internacional, especialmente a nivel demográfico y epidemiológico, y cómo se han adaptado los sistemas de salud.

En cuanto a las ciencias sociales en Chile, ha surgido el interés en relacionar salud y migración en diversas investigaciones; como el estudio de Cortez que pone en relieve la frágil situación de los inmigrantes en situación administrativa irregular, advirtiendo que uno de los aspectos de mayor impacto en el acceso a la atención médica, es la condición migratoria de las personas ${ }^{(21)}$. Asimismo, en otra investigación, Cortez et al. señalan que "aun cuando las personas tengan su situación migratoria regularizada, existe un alto porcentaje de no inscritos en el consultorio..."(22). Algunas investigaciones en el país han abordado aspectos más específicos relacionados a la salud sexual, como es el caso del estudio enfocado en los jóvenes inmigrantes realizado por Cortez en el que se presentan los riesgos y vulnerabilidades 
que tiene este grupo frente al $\mathrm{VIH} / \mathrm{sida}^{(23)}$. Siguiendo esa línea, el estudio de Núñez demuestra las dificultades que tienen las mujeres peruanas en el acceso y la atención en salud sexual y reproductiva, evidenciando las discriminaciones de las que son víctimas ${ }^{(24)}$.

Estos aportes generalmente señalan y tratan los problemas en forma de caracterización o denuncia. En cambio, en este artículo nos centraremos en analizar los criterios de acceso a la salud de los inmigrantes indocumentados, con el objetivo de abordar el origen de las dificultades que enfrentan en Chile. En segundo lugar, las investigaciones referidas ayudan a identificar la ausencia de información sobre situaciones conflictivas que viven los pacientes inmigrantes. Por tanto, abordaremos la relación que se establece entre los funcionarios administrativos, profesionales de la salud (en adelante funcionarios) y los pacientes inmigrantes, y estos últimos y el SPS. Presentaremos las percepciones de los funcionarios sobre los usuarios inmigrantes en general, con el fin de comprobar nuestra hipótesis. Planteamos que entre los funcionarios de salud y los usuarios inmigrantes existe una relación de poder basada en la desigualdad social (asimetría), política (nacionalismo) y cultural (alteridad). Nos apoyamos en estudios que demuestran que el espacio clínico es relacional y sobre todo complejo en un contexto pluralista, ya que el sentido de la vida, la muerte y el sufrimiento no son universales ${ }^{(25)}$. Cognet señala que la diferenciación cultural que establecen los terapeutas con relación a los pacientes extranjeros surge en la confrontación con la alteridad: "la cultura ya no es la de un otro lejano sino más bien de un otro en nuestra casa"(26). Por otra parte, Fassin ${ }^{(27)}$ explica que la "salud de los inmigrantes" es una preocupación de larga data, porque lleva el peso del estigma de la introducción de nuevas enfermedades, lo que representa un riesgo para la sociedad en su conjunto, bacteriológica y económicamente. No obstante, este mismo autor plantea que la relación entre la salud pública y las políticas de inmigración posiciona la cuestión del cuerpo y de la vida en los fundamentos de nuestra moral política, ya que las disparidades no se resumen a diferencias socioeconómicas, las brechas subsisten cuando se trata de poblaciones minoritarias, como los extranjeros ${ }^{(28)}$. Es así como la desigualdad social se manifiesta en lo que Caizzi llama una "relación de evitamiento y de dominación unilateral" y señala que es difícil que un agente de un servicio público no elabore su propio sistema de representaciones que puede impedir la finalidad prioritaria del servicio: el acceso a derechos sociales ${ }^{(29)}$. El resultado de esta desigualdad puede ser una discriminación, que en palabras de Fassin, "es un tratamiento desigual fundado en la aplicación de un criterio ilegítimo" ${ }^{\prime(30)}$.

Este artículo pretende llenar parte de los vacíos en la materia y aportar información documentada y actualizada sobre el problema del acceso a la salud de los inmigrantes indocumentados en Chile, a pesar de que puede afectar a la población inmigrante en general.

\section{METODOLOGÍA}

El presente texto está basado, principalmente, en los resultados de una investigación doctoral( ${ }^{(8)}$ y en el seguimiento que hemos realizado sobre el tema en nuestros proyectos actuales. Se trabajó una metodología cualitativa de investigación, inscrita en una etnografía del espacio clínico. Esto significa una inmersión del investigador en el terreno que se va a estudiar, para describirlo e interpretarlo mejor. Para lograrlo, se realizó, en 2009, un trabajo de campo de cuatro meses en los Centros de Salud No. 1, No. 5 y Los Nogales, así como en el Hospital San Borja Arriarán, dependientes del Servicio de Salud Metropolitano Central. Se realizaron 19 entrevistas a funcionarios representantes de cada una de las áreas de trabajo de los establecimientos (Tabla 2). Los directores de los centros indicaron a quién entrevistar en función de sus cargos y solicitaron su colaboración. Cada entrevistado firmó un consentimiento informado y autorizó el registro de 
Tabla 2. Número y rango etario de funcionarios de salud entrevistados, según cargo/ ocupación y sexo. Región Metropolitana, Chile, 2009.

\begin{tabular}{|c|c|c|c|c|}
\hline \multirow{2}{*}{ Cargo/ocupación } & \multirow{2}{*}{$\mathrm{n}$} & \multicolumn{2}{|c|}{ Sexo } & \multirow{2}{*}{$\begin{array}{l}\text { Rango } \\
\text { etario }\end{array}$} \\
\hline & & $\mathrm{H}$ & M & \\
\hline Directores de consultorios (médicos) & 3 & 2 & 1 & $25-35$ \\
\hline Asistentes sociales & 3 & 1 & 2 & $25-45$ \\
\hline Psicólogas & 2 & - & 2 & $25-35$ \\
\hline Enfermeras ( 4 jefas de sectores, 1 apoyo clínico) & 5 & - & 5 & $35-55$ \\
\hline Nutricionista & 1 & - & 1 & $45-55$ \\
\hline Médico (subdirector) & 1 & 1 & - & $45-55$ \\
\hline Coordinador administrativo & 1 & 1 & - & $25-35$ \\
\hline Funcionaria de Oficina de informaciones, reclamos y sugerencias & 1 & - & 1 & $35-45$ \\
\hline Técnico paramédico & 1 & 1 & - & $45-55$ \\
\hline Funcionaria del Servicio de orientación médica & 1 & - & 1 & $45-55$ \\
\hline
\end{tabular}

Fuente: Elaboración propia.

audio de las entrevistas. El número de entrevistas fue determinado por la saturación de la información recopilada; es decir, hasta no encontrar datos nuevos. Un método complementario fue la observación participante, que consiste en ser parte del grupo estudiado, asumiendo algún rol, con el fin de comprender desde su interior el funcionamiento del grupo. En nuestro caso, participamos de un equipo de trabajo para la elaboración y testeo de un proyecto piloto de capacitación y sensibilización sobre atención a migrantes dirigido a funcionarios del Servicio de Salud Metropolitano Central. Se realizaron dos intervenciones; un taller de 3 horas en el que participó la totalidad de los funcionarios del centro de salud No. 1 (aproximadamente 90 funcionarios) y una capacitación de 16 horas cronológicas, repartidas en dos sesiones, en la que participaron 60 funcionarios.

El análisis de las entrevistas transcritas y anotaciones de conversaciones y observaciones está basado en un análisis de las percepciones de los sujetos estudiados, en este caso los funcionarios. Tal como lo expresa Witker Barra $^{(31)}$, entendemos por percepción:

\begin{abstract}
...la actividad cognoscitiva que se relaciona con el almacenamiento y procesamiento de información y que está constituida por las siguientes habilidades: clasificar, seleccionar, simplificar, abstraer, analizar y sintetizar la información recibida. ${ }^{(31)}$
\end{abstract}

Las percepciones fueron diferenciadas en "apreciaciones", entendidas como consideraciones personales y comentarios; y "constataciones", entendidas como afirmaciones y certezas. Luego, se estableció una subcategoría para indicar el carácter subjetivo que otorga un juicio de valor "positivo", "negativo", "ambiguo" o "neutro" de los enunciados al referirse a los pacientes inmigrantes o a sus prácticas.

Para realizar este análisis se leyeron las entrevistas y anotaciones de campo, se seleccionaron las frases y conceptos de mayor aparición y frecuencia. Luego se interpretaron los relatos, buscando las relaciones y jerarquización de las percepciones. De esta manera nos dimos cuenta de que, unas veces, se trataba de una apreciación, es decir, de una opinión forjada a partir de las percepciones 
personales y, otras, de una constatación, o sea una afirmación que defendían.

Por otra parte, se estudió el marco legal chileno en materia de inmigración, así como la evolución de las normativas y políticas de salud focalizadas en la población indocumentada. Además, se llevó a cabo un monitoreo de la prensa nacional y el periódico local "La Estrella de Iquique", de la región de Tarapacá, seleccionando las noticias que tenían como palabras claves: inmigración, inmigrantes, peruanos, bolivianos. Esta información se utilizó para ilustrar la relación entre las políticas públicas, la acción de los funcionarios y las implicancias para los inmigrantes.

Esta investigación contó con el aval del Ministerio de Salud, del Fondo Nacional de Salud y del Servicio de Salud Metropolitano Central. Estas instituciones autorizaron el trabajo de campo, las pautas de entrevistas, y participaron en la elaboración y puesta en marcha del proyecto de intervención.

\section{ETNOGRAFÍA DEL ESPACIO CLÍNICO}

En esta sección presentamos los resultados del cruce de datos recopilados en el análisis de entrevistas, la observación participante y la revisión de notas publicadas por la prensa escrita.

\section{Relación funcionarios-pacientes inmigrantes}

Los Centros de Salud No. 1 y No. 5 de Santiago Centro (Chile) son viejos establecimientos, con una arquitectura sólida, muros espesos y altos techos que producen un microclima de varios grados por debajo de la temperatura exterior y una luminosidad de baja intensidad. Estos edificios vetustos no ayudan a los pacientes inmigrantes a sentirse acogidos, mucho menos a primera hora de la mañana, cuando los usuarios del centro - "la comunidad" - se precipitan para obtener un turno de atención médica. El personal de salud Ilama "la comunidad" solo a los usuarios nacionales; los inmigrantes son considerados como no-comunitarios y son llamados "nuevos usuarios". Es en este momento de la jornada que la mayor parte de los conflictos entre nacionales y extranjeros se develan y que se pueden observar fácilmente situaciones hostiles hacia los "nuevos usuarios".

La Figura 1 presenta las percepciones más nombradas en las entrevistas, conversaciones informales y observaciones sobre los pacientes inmigrantes en general. No encontramos tendencias asociadas a la posición social del funcionario, como el cargo o el nivel educacional, ni asociadas al grupo etario, ni al sexo del entrevistado. Encontramos que existe una predisposición por parte de los funcionarios, de manera transversal, a percibir negativamente a los pacientes inmigrantes (16 apreciaciones negativas de 32 apreciaciones y 10 constataciones negativas de 21 constataciones), por ejemplo: "son demandantes", "son más exigentes", "son policonsultantes". Estas tres percepciones están asociadas a que los pacientes inmigrantes presionan al SPS, esto se puede interpretar de la siguiente manera: "vienen por cualquier cosa, quieren que se les atienda bien y están a la defensiva". Esto lo hemos analizado con mayor detalle en otras publicaciones ${ }^{(8,9)}$.

Por otra parte, también se nombran ciertas características positivas, tales como: "están bien informados, conocen sus derechos", "cumplen los requisitos exigidos", "hablan bien". Estas tres percepciones dan cuenta de que los funcionarios reconocen que estos pacientes generan sus propias estrategias, personales y/o colectivas para acceder a la salud, a pesar de las barreras que se les imponen.

Encontramos además, en menor medida, percepciones ambiguas, es decir que no son explícitamente negativas ni positivas, como "tienen una forma particular de ser" y otras neutras, que no otorgan juicios de valor, como "se atienden más mujeres, embarazadas". A pesar de ser ambiguas o de parecer neutras, estas percepciones dejan entrever que, por un lado, se trata de una población "distinta" y que, por otro lado, esta población presiona al sistema desde el área de 


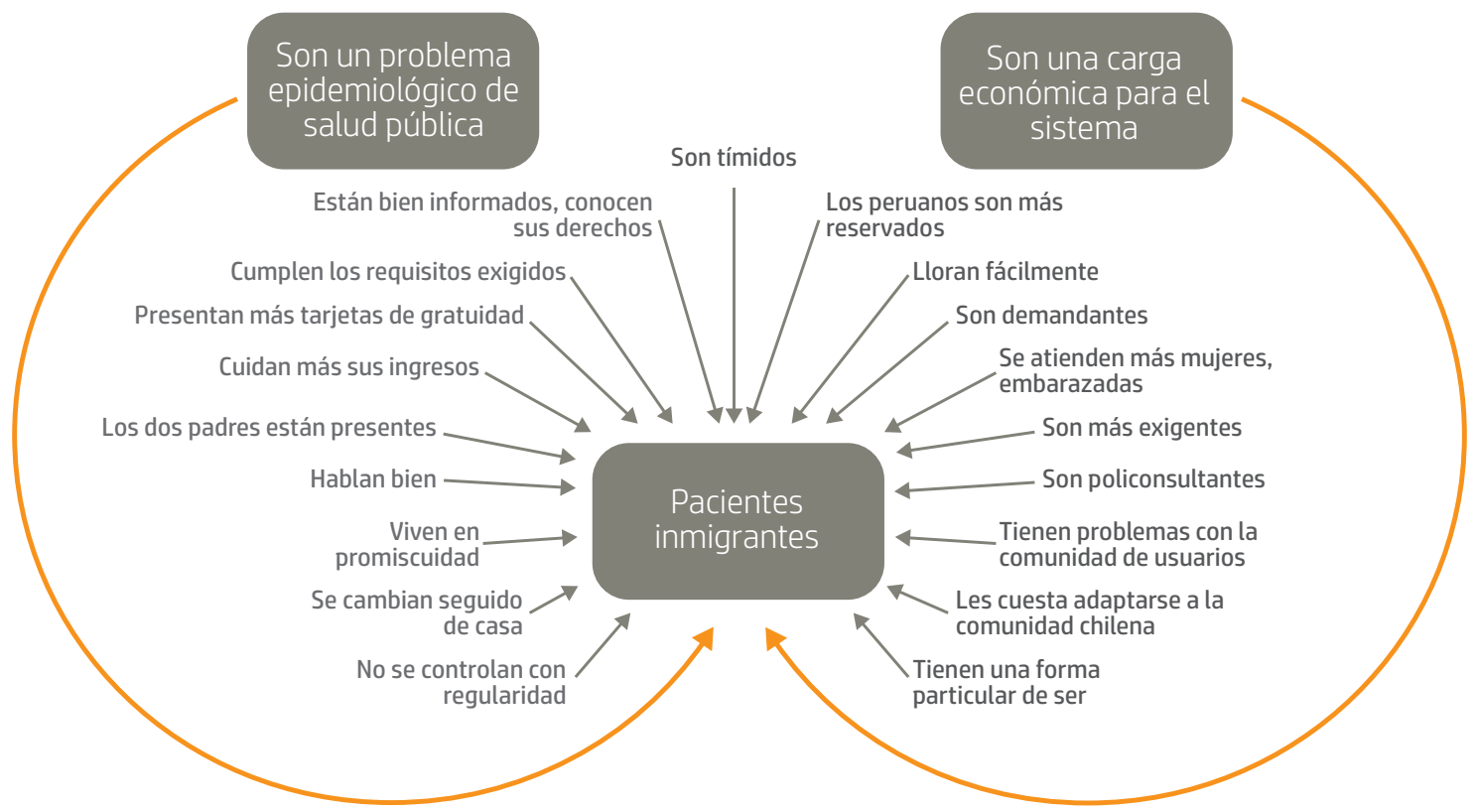

Figura 1. Percepciones de los funcionarios de salud sobre pacientes inmigrantes. Región Metropolitana, Chile, 2009.

Fuente: Elaboración Propia.

maternidad. Por último, hallamos que hay dos percepciones negativas más importantes y que son enunciadas como constataciones: las que indican que los inmigrantes "son un problema epidemiológico de salud pública" y las que señalan que "son una carga económica para el sistema". Estas percepciones influyen al momento del acceso a la salud y la atención médica, en particular, cuando preexisten otras barreras como la falta de documentación. Esta condición implica que, por ejemplo, una usuaria no puede ser contabilizada en el sistema, ya que no puede ser inscrita al no contar con un número de identificación nacional. Esto implica que el sistema no recibe la subvención del FONASA por las personas no inscritas, ya que el financiamiento del SPS se organiza en función del número de inscritos, es decir, per capita: por cada inscrito, los establecimientos reciben una subvención. A continuación veremos cómo esto va a influir en la violación de los principios más básicos del sistema de salud.

\section{Relación Sistema Público de Salud- pacientes inmigrantes indocumentados}

Los datos indican que las dificultades de acceso de los inmigrantes indocumentados se ven reforzadas por dos aspectos: 1) el incumplimiento de los acuerdos ministeriales y 2) el criterio personal de los agentes.

Al indagar sobre la aplicación de los acuerdos interministeriales que extienden la cobertura en salud a los "irregulares", los datos revelan el incumplimiento de estos. Particularmente, en la prensa escrita se detectaron casos en los que se cuestiona el acceso de esta población al SPS. Por ejemplo, el de una mujer que permaneció retenida cinco días en el Hospital de Arica por supuesta deuda del parto; según instituciones y agrupaciones de inmigrantes de la región esta no es una situación aislada. Así lo publicó el medio digital El Morrocotudo, en el titular del 17 septiembre de 2014: "Organizaciones de migrantes denuncian supuesto caso de discriminación en frontera Chile-Perú"(32). Por su parte, el 
Instituto Nacional de Derechos Humanos (INDH), afirma que en el Hospital de Tarapacá la entrega del certificado de nacimiento de los hijos de inmigrantes está sujeta al pago del parto. En muchos casos, al no contar con este dinero, las madres se escapan del establecimiento sin el certificado, lo que impide la inscripción de los niños en el Registro Civil. La situación indocumentada de los niños los deja sin acceso a derechos sociales. El INDH reporta casos documentados, a partir de los cuales afirma: "estas situaciones podrían constituir infracciones a la legislación vigente" ${ }^{\prime \prime(3)}$

Desde el año 2006, aparecen noticias en la prensa en las que se cuestiona la atención a los inmigrantes en el SPS. La zona fronteriza es particularmente sensible al tema. Con el titular "Las pérdidas en salud con los extranjeros" ${ }^{\prime \prime(34)}$ la directora del Servicio de Salud Arica cuenta cómo aumentan las atenciones de extranjeros sin previsión que no pueden pagar las prestaciones en la Unidad de Urgencia (15 mil pesos por persona, equivalente a 22 dólares) y el Servicio de Maternidad (mínimo de 150 mil pesos, equivalente a 220 dólares) del Hospital Dr. Juan Noé de Arica, y señala: "A nosotros no nos llegan recursos extras por ser una ciudad bifronteriza y por atender pacientes extranjeros. Son solo recursos propios que van en desmedro de otras atenciones"(34). Por su parte, el director de Salud Municipal de lquique (Región de Tarapacá) indicó en la Escuela de Gestores en Salud Migrante, organizada por el MINSAL en la ciudad de lquique el 11 de diciembre de 2014, que el "desfinanciamiento" del SPS se debía a la alta demanda de los inmigrantes sin previsión (información registrada en nuestras notas de campo).

Asimismo, se constata que no se asegura una política de difusión de los acuerdos, ni de capacitación dirigida a los agentes, simplemente se deja que cada Servicio de Salud aplique su propia política hacia esta población. El Oficio ORD-A-14 № 3229(35), establece:

Para dar fiel cumplimiento a estas disposiciones ministeriales, los directores de los Servicios de Salud deberán difundir estas medidas a todo el personal bajo su responsabilidad y coordinarse con los encargados de las oficinas de Extranjería y Migración correspondiente a su jurisdicción territorial.

Siguiendo este mandato, el Servicio de Salud Metropolitano Central distribuyó una minuta sobre el abordaje que los funcionarios deben tener hacia los inmigrantes en todos sus establecimientos. La minuta menciona "diferentes categorías de inmigrantes", el objetivo es que los funcionarios inciten a los indocumentados a tramitar su regularización y llama a aplicar un "criterio personal", según el caso, proponiendo dar un trato excepcional a estos usuarios, sobre la base del respeto de los derechos humanos, pero respondiendo al mandato del MINSAL de protección de la salud pública. El documento dice: "Existe un problema epidemiológico que en aras del bien común el Estado tiene que controlar"(36). El inconveniente surge al presentar a esta población como un "problema" en un documento que no es explicado en una capacitación, sino solamente distribuido. Esto provoca en los funcionarios una percepción negativa hacia pacientes pertenecientes a este grupo.

Si analizamos lo que sucede con el Decreto de Ley 1094 de 1975, Ilamada Ley de Extranjería y Migraciones (que regula la inmigración en Chile), en el caso de entrega de visas, estipula aplicar un "criterio discrecional". Esto significa que los criterios se dividen entre situaciones en las que se debe y otras en las que se puede prohibir el ingreso, rechazar una visa y revocar una visa. Este mismo criterio discrecional, aplicado al caso estudiado, ha producido discriminaciones en el acceso a la salud de inmigrantes. Destacamos dos casos ilustrativos.

Caso 1: En el año 2008 una mujer peruana, en su desesperación, tras esperar casi dos horas para ser ingresada a sala de parto del Hospital San José, acude al baño del establecimiento donde da a luz a su hijo. Esta escena fue registrada por su pareja con un teléfono celular y es difundida ampliamente por la prensa. EI 
padre del bebé interpuso una demanda por negligencia al hospital, ya que declaró sentirse "maltratado y ultrajado," por no recibir la atención pertinente. Así fue publicado por el periódico digital El Mercurio Online el 9 de abril de 2008, bajo el titular "Mujer da a luz en baño de hospital San José"(37).

Caso 2: El 7 de octubre de 2014 muere un lactante de padres bolivianos, el cual en horas de la madrugada había presentado un cuadro diarreico y según la denuncia de sus progenitores, el pequeño no recibió la atención oportuna. Hay diversas reacciones, entre las que destaca la del INDH que declara: “Un bebé de nueve meses, de padres bolivianos, falleció en Arica por no recibir atención médica", noticia publicada por el Diario Uchile, el 10 de octubre de 2014, bajo el titular "INDH condena a salud pública por muerte de lactante" ${ }^{\prime(38)}$. El motivo de la negación de la atención es económico, "El Hospital Juan Noé no los habría atendido porque no tenían los 30 mil pesos que se les cobraba", información publicada por Biobiochile, el 8 de octubre de 2014, bajo el titular "Investigan si Hospital de Arica negó atención a bebé boliviano que murió en Azapa"(39).

\section{Pacientes ilegítimos}

La relación entre paciente y profesional de la salud es siempre asimétrica. Quienes han estudiado la problemática plantean que la relación paciente-terapeuta es una relación social, e incluso que las relaciones clínicas son relaciones de poder ${ }^{(25)}$. En nuestro estudio observamos que, en la relación paciente inmigrante/funcionario de salud, esta condición se observa con mayor contraste, ya que el inmigrante representa la alteridad, es decir, el "otro," la persona que se encuentra más allá de la frontera del "nosotros" (comunidad nacional). Por esta razón, los funcionarios cuestionan el acceso a la salud de los inmigrantes a partir de la diferenciación que se asigna según el estatus jurídico o administrativo, y según las prácticas o cualquier característica definida como cultural o física, etc.
La principal consecuencia es que se produce desigualdad en salud entre nacionales y extranjeros. La connotación de la denominación "nuevos usuarios" en contraposición a una "comunidad de usuarios", es determinante y muestra desde el inicio la separación establecida entre "los que son de aquí" y "los que llegan de afuera". La condición de alteridad de estos pacientes y la asimetría en las relaciones de poder surgen como un arma de defensa de los intereses del "nosotros". Así, la nacionalidad, el estatus migratorio y la capacidad económica aparecen como criterios subjetivos (justificación simbólica) para decidir quién es paciente legítimo del SPS y quién no. Sucede esto a pesar de que, desde la perspectiva del derecho, la noción de paciente ilegítimo no se sostiene, al existir el compromiso normativo de acceso a la salud a ciertas categorías de inmigrantes indocumentados.

En el caso de la mujer embarazada, su estatus migratorio es irrelevante, ya que se evidencia una predisposición a no atender la urgencia. En el caso del lactante, si se trata de indocumentados, prevalece la denegación de la atención de urgencia por el no pago. Los padres de este niño son inmigrantes bolivianos, indígenas e indocumentados, razón por la cual los funcionarios proponen la atención privada.

Se constata que, en el ámbito del acceso a la salud de los indocumentados, la desigualdad en salud es mayor, debido a que los acuerdos interministeriales para dar atención médica a ciertos grupos de indocumentados no siempre se cumplen. Al ser considerados una carga económica para el SPS, los funcionarios exigen el pago de una atención privada, desconociendo los acuerdos. Vemos que el criterio que se aplica depende del funcionario y en ningún caso de la normativa. Lesselier utiliza la noción de "infra derecho," utilizado para resolver situaciones complejas y coyunturales: "una gran parte del estatus de extranjeros/as está regida por lo que se llama el 'infra derecho': el del mesón de atención, de la circular y del poder discrecional de las autoridades administrativas"(40).

Por su parte, Bec afirma que la desigualdad es una relación social que promueve la 


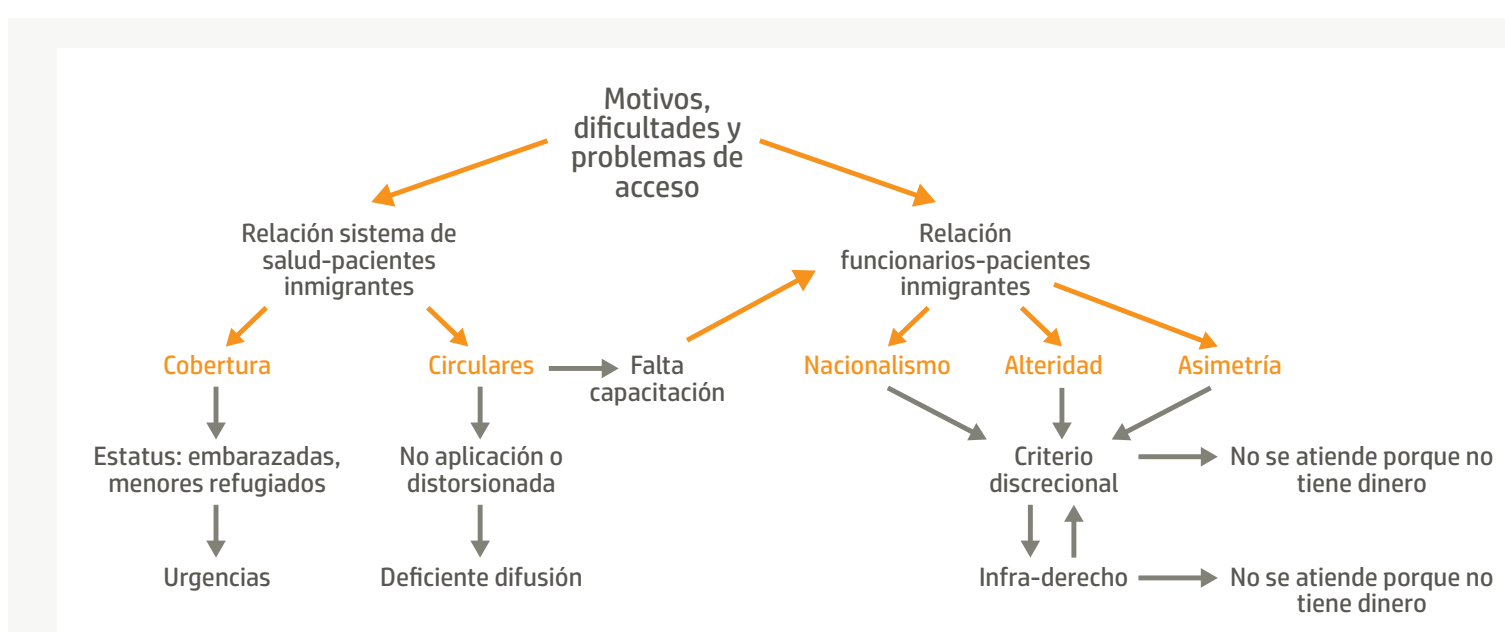

Figura 2. Mapa de articulación conceptual de resultados.

Fuente: Elaboración propia a partir de Liberona Concha ${ }^{(8)}$.

pobreza, "razonar en términos de desigualdad es razonar sobre una realidad en cuyo centro se despliegan interdependencias entre los individuos y los grupos sociales". La autora asocia la pobreza al funcionamiento mismo de la sociedad; "en el cruce a la vez de trayectorias individuales y mecanismos económicos y sociales"(41). Una manera complementaria de observar y describir la desigualdad, es la que considera una distinción entre dos órdenes de cosas: por un lado, la desigualdad es productora de diferencias $y$, por otro, las diferencias reales o supuestas "soportan" la desigualdad. La mantención de esta distinción permitiría articular lo cultural con lo social, lo económico y lo político, provocando relaciones variables que podrían ser analizadas como relaciones interétnicas o "interraciales"(42). Por tanto, para el caso estudiado, podemos afirmar que el acceso a la salud de los inmigrantes indocumentados en Chile está basado en estas relaciones interétnicas desiguales (Figura 2).

Pero, hay otro elemento a considerar y es que una de las principales percepciones de los funcionarios con respecto a los pacientes inmigrantes es que representan una carga económica para el sistema. Esta situación está directamente relacionada al hecho de que FONASA no otorga una subvención extra para la atención que autoriza y promueve la normativa. Por tanto, la presión que produce la falta de recursos, conjugada con la poca o nula capacitación en cuanto a derechos, se presenta como el escenario perfecto para que la discriminación opere con amplia legitimidad. Siguiendo esta lógica, se puede afirmar que los acuerdos interministeriales que producen reformas legales, consensuadas a nivel político-normativo, al no ser acompañadas de los recursos y/o de la planificación adecuada para su implementación en el nivel ejecutivoadministrativo, son la principal causa del problema evidenciado en esta investigación.

\section{CONCLUSIONES}

Las dificultades que tienen los inmigrantes indocumentados para acceder a la atención médica en Chile se ven afectadas por la percepción de los funcionarios de salud quienes, al elaborar su propio sistema de representaciones, los ven como una carga para el sistema, y no como parte legítima de la comunidad de usuarios. Los datos expuestos respaldan la idea de que tanto el infraderecho, como la discriminación hacia el grupo que representa la alteridad en el sistema, se 
producen indistintamente de la posición social del funcionario, no dependen del cargo, ni del nivel educacional, ni de la edad, ni del sexo, sino de la xenofobia y los prejuicios derivados de las nociones nacionalistas.

Esas percepciones negativas, junto con las facultades discrecionales ejercidas por los funcionarios de salud, podrían invalidar derechos consagrados en la legislación chilena, o contemplados en tratados internacionales y acuerdos interministeriales. Esta situación, además, puede generar mayor pobreza a los indocumentados que no tienen otra opción que la salud privada.

Sin embargo, esta investigación también revela que gran parte de la responsabilidad recae en quienes definen la normativa y no consideran recursos adicionales, ni elaboran una política nacional que asegure la difusión de los acuerdos, así como capacitaciones adecuadas y sensibilización pertinente para los funcionarios de salud. Por tanto, se trataría de una discriminación institucional y no solo individual del funcionario, lo que abre ventanas a análisis adicionales, que esperamos abordar en futuras investigaciones.

Estas conclusiones tienen implicaciones, tanto para el desarrollo de la investigación en la materia, como para la práctica profesional y las políticas de salud focalizadas. Al aportar datos empíricos, futuros estudios pueden usarlos para observar los avances o retrocesos en la aplicación de las políticas hacia este grupo. Asimismo, puede ser referencia de estudios especializados en las teorías de las relaciones interétnicas. En cuanto a la práctica en salud, pretendemos que este trabajo sirva para que los profesionales de la salud puedan ponerse en el lugar del otro y repensar su rol en la relación social paciente-terapeuta. Por último, permite visualizar problemas de índole relacional, en los cuales los individuos son los que toman decisiones por sobre los marcos jurídicos. Esto podría ayudar a comprender por qué persisten trabas ahí donde se han diseñado soluciones.

\section{AGRADECIMIENTOS}

Investigación financiada por la Comisión Nacional de Investigación Científica y Tecnológica, a través del Proyecto de Inserción de Capital Humano en la Academia 2014 No 79140078 "Relaciones interétnicas y transfronterizas en el marco de las migraciones internacionales actuales". Asimismo, agradecemos a la Vicerrectoría de Investigación, Innovación y Postgrado de la Universidad Arturo Prat por el financiamiento a un Congreso Internacional, que dio como resultado este artículo.

\section{REFERENCIAS BIBLIOGRÁFICAS}

1. Ministerio de Desarrollo Social. Encuesta de Caracterización Socioeconómica Nacional: Contenidos: Inmigrantes [Internet]. 2013 [citado 1 mar 2016]. Disponible en: https://tinyurl.com/ y7v68npb.

2. Texidó E, Gurrieri J. Panorama migratorio de América del Sur 2012. Buenos Aires: OIM; 2012.
3. Departamento de Extranjería y Migración, Ministerio del Interior y Seguridad Pública. Migración en Chile 2005-2014 [Internet]. 2016 [citado 10 mar 2016]. Disponible en: https://tinyurl.com/ grb856c.

4. Departamento de Extranjería y Migración, Ministerio del Interior y Seguridad Pública. Caracterización de la migración en chile: Una Mirada desde instrumentos gubernamentales [Internet]. 2014 [citado 10 mar 2016]. Disponible en: https:// tinyurl.com/ybofpmew.

5. Castles S. Migración irregular: causas, tipos y dimensiones regionales. Migración y Desarrollo. 2010;8(15):49-80.

6. República de Chile, Presidenta. Instructivo Presidencial $N^{\circ}$ 9, Política Migratoria en Chile. Santiago: 2008.

7. Naciones Unidas. Convención internacional sobre la protección de los derechos de todos los trabajadores migratorios y de sus familiares [Internet]. 1990 [citado 10 mar 2016]. Disponible en: https://tinyurl.com/yakn8akk. 
8. Liberona Concha N. La prise en charge des immigrants sud-américains dans le système public de santé chilien: un espace de tensions interethniques. [Tesis de doctorado]. Paris: Université Paris Diderot, Paris 7; 2012.

9. Liberona Concha N. De la alterisación a la discriminación en un sistema público de salud en crisis: conflictos interétnicos a propósito de la inmigración sudamericana en Chile. Revista de Ciencias Sociales. 2012;(28):19-38.

10. Organización Mundial de la Salud. Migración internacional, salud y derechos humanos. Ginebra: OMS; 2005.

11. Centro Nacional para la Prevención y el Control del VIH/SIDA. SIDA: Aspectos de salud pública. México: CENSIDA/INSP; 2006.

12. Molina-Salas Y, De las Mercedes LomasCampos M, Romera-Guirado FJ, Romera-Guirado MJ. Influencia del fenómeno migratorio sobre la tuberculosis en una zona semiurbana. Archivos de Bronconeumología. 2014;50(8):325-331.

13. AIDS \& Mobility. Sida y movilidad: Situación, políticas e intervenciones (Informe reunión satélite). XIV Conferencia Internacional del Sida. Barcelona: AIDS \& Mobility; 2002.

14. Arredondo López A, Orozco Núñez E, Wallace S, Rodríguez M. Indicadores de gobernanza para el desarrollo de estrategias binacionales de protección social en la salud de los migrantes. Saúde e Sociedade. 2013;22(2):310-327.

15. Jelin E. (dir.). Salud y migración regional: ciudadanía, discriminación y comunicación intercultural [Internet]. Buenos Aires: IDES; 2005 [citado 10 mar 2016]. Disponible en: https://tinyurl.com/ y8ee9p27.

16. Jelin E, Grimson A, Zamberlin N. ¿Servicio?, ¿Derecho?, ¿Amenaza? La llegada de inmigrantes de países limítrofes a los servicios públicos de salud. In: Salud y migración regional: ciudadanía, discriminación y comunicación intercultural [Internet]. Buenos Aires: IDES; 2005 [citado 10 mar 2016]. Disponible en: https://tinyurl.com/ y8ee9p27.

17. Waldman TC. Movimentos migratórios sob a perspectiva do direito à saúde: imigrantes bolivianas em São Paulo. Revista de Direito Sanitário. 2011;12(1):90-114.

18. Dal Prá KR, Mendes JMR, Mioto RCT. O desafio da integração social no MERCOSUL: uma discussão sobre a cidadania e o direito à saúde. Cadernos de Saúde Pública. 2007;23(Sup 2):S164-S173.
19. Demoscópica. Diagnóstico y factibilidad global para la implementación de políticas locales de salud para inmigrantes de la zona norte de la Región Metropolitana [Internet]. 2009 [citado 10 mar 2016]. Disponible en: https://tinyurl.com/ y83nbc9d.

20. Vásquez-de Kartzow R. Impacto de las migraciones en Chile: Nuevos retos para el pediatra, ¿estamos preparados? Revista Chilena de Pediatría. 2009;80(2):161-167.

21. Cortéz Salas A. Migración internacional: un desafío para las políticas públicas de Chile. In: Niños y niñas migrantes: Políticas públicas, integración e interculturalidad Santiago: Colectivo sin Fronteras; 2007. p. 105-118.

22. Cortéz Salas A, Reyes GC, Reyes MS. Salud sexual y reproductiva y migración latinoamericana en Chile: Una aproximación cualitativa a las situaciones de riesgos y conductas de prevención del VIH-SIDA. Cuadernos Médico Sociales. 2010;50(4):322-339.

23. Cortéz Salas A, Donoso Orellana C, Loredo Chupán P. Diagnóstico de riesgo y vulnerabilidad frente al VIH/SIDA de jóvenes inmigrantes peruanos. Santiago: Colectivo sin Fronteras; 2007.

24. Núñez N, Torres C. Mujeres migrantes peruanas y salud reproductiva: Usuarias de consultorios de salud de la zona norte de la Región Metropolitana. Santiago: Instituto de la Mujer; 2007.

25. Fortin S, Laprise E. L'espace clinique comme espace social. In: Cognet M, Montgomery C. Éthique de l'altérité: La question de la culture dans le champ de la santé et des services sociaux. Montréal: Les Press I'Université Laval; 2007. p.191-214.

26. Cognet $M$. Au nom de la culture: le recours à la culture dans la santé. In: Cognet $M$, Montgomery $\mathrm{C}$. Éthique de l'altérité: La question de la culture dans le champ de la santé et des services sociaux. Montréal: Les Press I'Université Laval; 2007. p. 39-64

27. Fassin D. Repenser les enjeux de santé autour de l'immigration. Hommes \& Migrations. 2000;(1225):5-12.

28. Fassin D. Santé et immigration: Les vérités politiques du corps. Cahiers de I'Urmis. 1999;(5):6976.

29. Caizzi A. Présence et représentation des usagers aux guichets des caisses d'Allocations familiales. Informations Sociales. 2010;(158):112-120. 
30. Fassin D. Discrimination et santé: enjeux politiques et signification sociale. Profession Banlieue. 2002;4(11):1-22.

31. Witker Barra I. Bolivia 2003: percepciones de la crisis en la prensa chilena y su impacto en la seguridad subregional y relaciones bilaterales. Santiago: Academia Nacional de Estudios Políticos y Estratégicos, Ministerio de Defensa Nacional; 2005.

32. Denuncia ciudadana: Mujer migrante lleva 5 días sin poder salir del Hospital de Arica hasta que pague el parto. El Morrocotudo [Internet]. 2014 [citado 10 mar 2016]. Disponible en: https:// tinyurl.com/krh8lvu.

33. Instituto Nacional de Derechos Humanos. Informe Misión de observación situación de la población migrante Iquique y Colchane. Santiago: INDH; 2013.

34. Cousins P. Las pérdidas en salud con los extranjeros. La Estrella de Arica [Internet]. 2006 [citado 10 mar 2016]. Disponible en: https:// tinyurl.com/y8gg2tsc.

35. Gobierno de Chile, Ministerio de Salud. Oficio ORD-A-14 No 3229 [Internet]. 2008 [citado 10 mar 2016]. Disponible en: https://tinyurl.com/ yax7luay.
36. Gobierno de Chile, Ministerio de Salud, Servicio de Salud Metropolitano Central. Minuta Atención Inmigrantes. Santiago: SSMC; 2008.

37. Mujer da a luz en baño de Hospital San José. El Mercurio Online [Internet]. 2008 [citado 10 mar 2016]. Disponible en: https://tinyurl.com/yajswqsx.

38. González T. INDH condena a salud pública por muerte de lactante. Diario Uchile [Internet]. 2014 [citado 10 mar 2016]. Disponible en: https:// tinyurl.com/y75zao4b.

39. Mardones C. Investigan si Hospital de Arica negó atención a bebé boliviano que murió en Azapa. Bibiochile.cl [Internet]. 2014 [citado 10 mar 2016]. Disponible en: https://tinyurl.com/ y98gv7jl.

40. Lesselier C. Politiques d'immigration en France: appréhender la dimension de genre. Les Cahiers du CEDREF. 2008;(16):189-208.

41. Bec C. Les pauvres font bande à part. Déviance et Société. 2002;26(2):245-251.

42. De Rudder V, Poiret C, Vourc'h F. L'inégalité raciste: I'universalité républicaine à l'épreuve. Paris: Presses Universitaires de France; 2000. 